\title{
Multi-axial fatigue behaviour of titanium periodic cellular structures produced by Selective Laser Melting (SLM)
}

\author{
Khalil Refai ${ }^{1, *}$, Charles Brugger ${ }^{1}$, Marco Montemurro ${ }^{1}$, and Nicolas Saintier ${ }^{1}$ \\ ${ }^{1}$ Arts et Métiers ParisTech, I2M CNRS UMR 5295, F-33400 Talence, France
}

\begin{abstract}
Additive manufacturing techniques such as selective laser melting (SLM) allow for manufacturing periodic porous titanium structures having complex geometry. The mechanical properties of these structures with different elementary cell (ECs) designs have been already studied in literature. However, their fatigue behaviour is not yet well understood. This work aims at proposing a numerical approach to predict the periodic cellular structures fatigue behaviour under multiaxial loadings. The approach is based on an explicit description of the EC combined to an extreme statistical analysis making use of a fatigue indicator parameter to investigate (and compare) different EC configurations. On the other hand, the numerical model relies on the use of a general numerical homogenisation scheme to properly apply the multi-axial loading conditions to the EC at the mesoscopic scale. Results show that lattice fatigue strength is strongly affected by the relative density as well as by the geometrical features of the EC.
\end{abstract}

\section{Introduction}

In the last two decades, a strong effort has been put on the development of periodic cellular structures fabricated by means of additive layer manufacturing (ALM) technology because of the advantages they offer including design freedom, high precision, and the ability to produce parts directly from a numerical model. Cellular structures exhibit a unique combination of properties, including lightness, high stiffness and high strength. The macroscopic elastic and fatigue behaviours of these structures depend on several factors such as elementary cell (EC) topology, material and manufacturing process characteristics, as well as loading conditions. By modifying the EC topology and the relative density at the mesoscopic scale, one can fulfill the desired requirements at the macroscopic one. Only few studies focusing on the fatigue behaviour of these structures which aim at understanding the relationship between the EC geometrical parameters and its fatigue strength are available in literature [1-3]. The determination of the fatigue behaviour of cellular structures is a costly and time consuming process since the range of possible EC and load type is almost infinite. Moreover, as mentioned before, there are many factors affecting it. Consequently, it is necessary to propose dedicated numerical approaches in order to investigate the influence of each factor on the macroscopic behaviour of the structure before manufacturing and experimental evaluation of the selected cell structure. Accordingly, the aim of this work is to provide a deeper insight into the influence of the EC topology and its relative density on cellular structure high cycle fatigue

*e-mail: khalil.refai@ensam.eu 
strength under multiaxial loadings. The paper is organised as follows. Material and cells geometries of the analysed cellular structures are presented in Section 2 . The general numerical strategy used to investigate the fatigue strength of the EC together with the multi-axial fatigue criterion employed in this work and the fundamentals of the extreme value theory (EVT) are presented in Section 3, whilst results are illustrated and discussed in Section 4. Finally, Section 5 ends the paper with some conclusions.

\section{Material and cells geometries}

This work aims to predict and compare the fatigue strength of various periodic cellular structures made of Ti-6Al-4V gathered in three main classes. The "lattice" class includes 18lattice structures whose EC is an arrangement of struts, as show in Fig. 1 [4]. "Thin-walled TPMS" and "Skeletal TMPS" classes include ECs obtained from triply periodic minimal surfaces (TPMS). The 9 "Thin-walled TPMS" (Fig. 2) [5] are generated by applying a constant thickness to an implicit surface. The 6 "Skeletal TMPS" structures (Fig. 3) are built by boolean operations on ECs from the other two classes. The behaviour of the bulk material (Ti-6Al-4V) composing the EC is supposed to be isotropic and purely elastic with the following properties: $E_{s}=110 \mathrm{GPa}$ and $v_{s}=0.34[4]$.

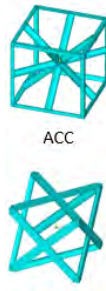

FCC
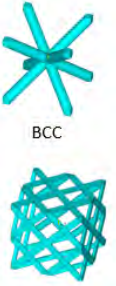

$\mathrm{FCC} 2+$

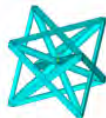

BFCC

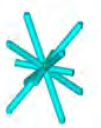

Octahedral

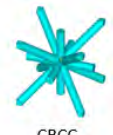

$\mathrm{CBCC}$

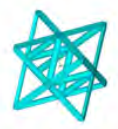

Octet
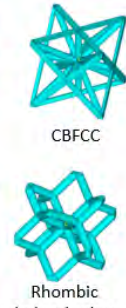

dodecahedron

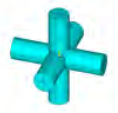

CC

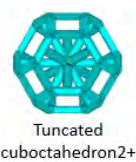

cuboctahedron 2
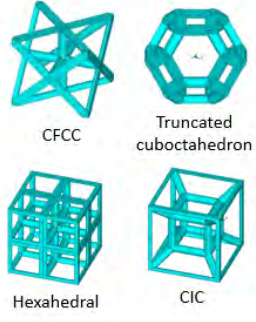

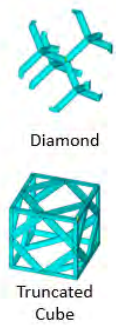

Fig. 1. Lattice structures.
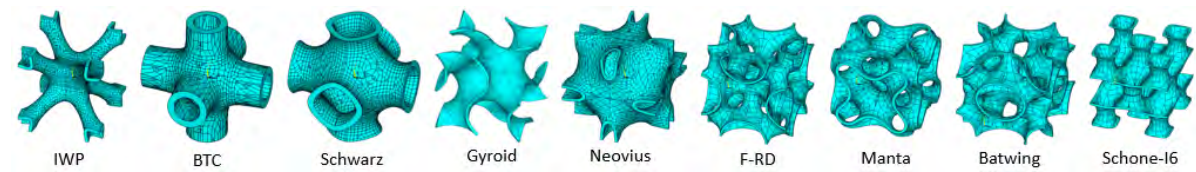

Fig. 2. Thin-walled TPMS structures.
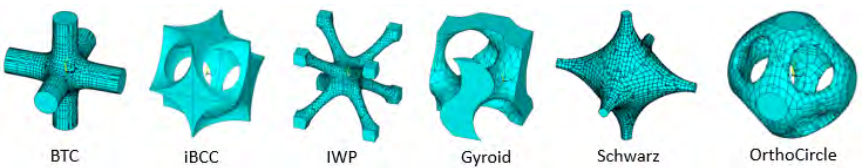

Fig. 3. Skeletal-TPMS structures.

\section{Numerical Strategy}

The flow-chart of the numerical strategy developed in this work is illustrated in Fig. 4. The numerical scheme combines an homogeneisation step in order to evaluate the macroscopic 
anisotropic elastic behavior of the EC, a finite element computation to access the cyclic local stress field in the EC, a fatigue post-processing procedure at each integration point of the EC and, finally, an extreme value-based statistical approach. The FE model of the EC and the related boundary conditions (BCs) are described in Section 3.1, whilst the fundamentals of the Crossland's criterion and of the EVT are given in Sections 3.2 and 3.3.
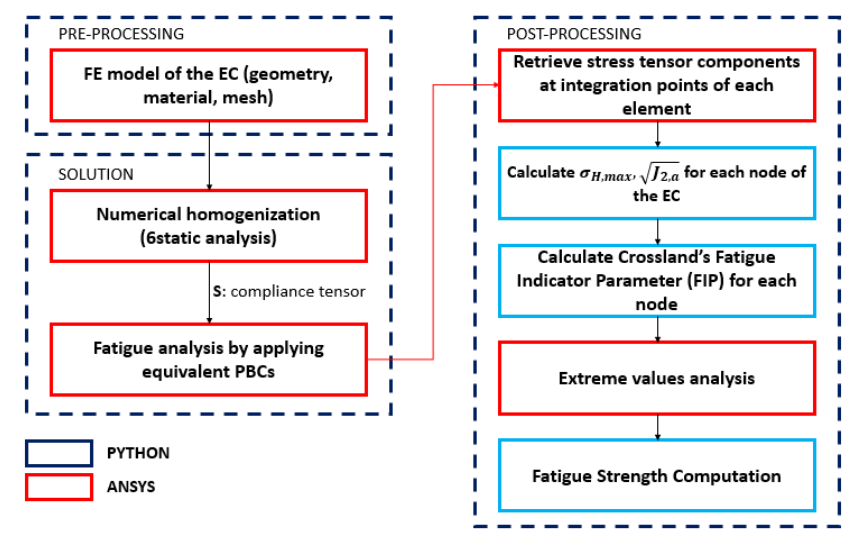

Fig. 4. Numerical procedure

\subsection{Finite element model of the elementary cell}

The FE model of the EC has been created within the commercial FE code ANSYS ${ }^{\circledR}$. This model is generated through an ad-hoc script in which the mesh has been properly related to the relevant parameters of the EC to take into account for different geometries. The EC is modelled by means of 20-nodes solid elements (SOLID186). A FE model made of solid elements is needed to provide a realistic representation of the EC geometry and to accurately estimate the 3D stress field generated inside the EC and which strongly influences its macroscopic elastic behaviour.

\subsubsection{Boundary conditions for the homogenisation analysis}

At the mesoscopic scale, the EC of the lattice structure can be interpreted, from a mechanical point of view, as an heterogeneous medium composed of two phases, i.e. the bulk material and the void. Conversely, at the macroscopic scale it can be modelled as an equivalent homogeneous anisotropic continuum whose mechanical response is described by a set of effective (or equivalent) material properties. The set of effective elastic properties of the EC can be determined through a suitable numerical homogenisation technique. Indeed, although the bulk material constituting the lattice is isotropic, its macroscopic behaviour (i.e. after homogenisation) can be anisotropic because the effective elastic properties depend upon the EC topology which strongly affects the stiffness tensor of the homogeneous material at the upper scale by introducing some preferential orientations (according to the symmetries of the EC). The effective properties of the EC are determined using the strain energy homogenisation technique of periodic media [6]. This homogenisation scheme has proven to be an efficient numerical procedure able to determine the equivalent properties of different heterogeneous materials characterised by complex EC topologies. The strain energy homogenisation technique of periodic media based on volume averaged stresses has already been utilised in other works, see [7, 8]. The detailed procedure can be found in [4]. 


\subsubsection{Boundary and loading conditions for the fatigue analysis}

The high-cycle fatigue analysis considered in this study assumes that both EC macroscopic and mesososcopic constitutive behaviours are always in the elastic domain. Only proportional loadings are considered here so that no principal stress directions rotations occur during the cyclic loading. The fatigue analysis is carried out on the EC at the mesoscopic scale by applying macroscopic cyclic loads $\Sigma_{0}(t)$ through an equivalent strain field at the mesoscopic scale of the EC.

The fatigue strength curve of the EC has been determined by considering five loading conditions at the macroscopic scale:

$$
L_{1}: \sigma_{0}(t), L_{2}: \tau_{0}(t), L_{3}: \tau_{0}(t)=\sigma_{0}(t), L_{4}: \tau_{0}(t)=\frac{\sigma_{0}}{3}(t), L_{5}: \tau_{0}(t)=3 \times \sigma_{0}(t) .
$$

The equivalent strain field to be applied to the EC at the mesoscopic scale (which corresponds to a specific cyclic stress state $\boldsymbol{\Sigma}_{0}(t)$ at the upper scale) has been introduced by means of periodic boundary conditions (PBCs) using the compliance tensor $\mathbf{S}=\mathbf{C}^{-1}$ determined after numerical homogenisation.

The results (in terms of displacement, strain and stress fields), provided by the fatigue analysis conducted on the mesoscopic FE model of the EC, have been post-processed by means of a suitable numerical procedure wherein the ANSYS ${ }^{\circledR}$ FE model has been interfaced with inhouse routines (programmed in PYTHON environment). It was observed that a mesh size of $0.05 \mathrm{~mm}$ for a cell size equal to $3 \mathrm{~mm}$ is sufficient to properly evaluate the EC fatigue strength.

\subsection{Multiaxial high-cycle fatigue criterion}

Fatigue criteria available in the literature can be classified into three families: (i) phenomenological criteria based on tensor invariants [9], (ii) criteria based on the definition of the critical plane in the space of stress / strain tensor components [10], (iii) energetic criteria [11]. The criterion considered here is the Crossland's criterion which belongs to the first class. The proposed methodology could be similarlly developped with other criteria. Crossland's criterion is defined as a linear combination of the maximum hydrostatic stress $\sigma_{H \text {,max }}$ over a cycle and of the amplitude of the second invariant of the deviatoric part of the stress tensor $\sqrt{J_{2 a}}$, i.e.

$$
\sqrt{J_{2 a}}+\alpha \sigma_{H, \max }<\beta,
$$

where $\alpha$ and $\beta$ are two parameters depending upon material and process. In this study, these parameters were taken from the work of Vayssette et al. [12] for Ti-6Al-4V machined specimens subjected to Hot Isostatic Pressing (HIP), manufactured by selective laser melting (SLM) and determined at $2.10^{6}$ cycles ( $\alpha=0.86$ and $\left.\beta=442.7 \mathrm{MPa}\right)$.

\subsection{Extreme Values Theory}

The stress and strain fields in the ECs (at the mesoscopic scale) are strongly heterogeneous. In addition, their gradients are substantial, due to small dimensions. In such cases, using only local (nodal) values to evaluate the fatigue strength usually is inadequate. The EVT framework is chosen to overcome this issue. The proposed approach is based on the extreme value distribution of a fatigue indicator parameter (FIP) related to the Crossland's criterion:

$$
F I P=\sqrt{J_{2 a}}+\alpha \sigma_{H, \max } .
$$


Let $\Omega_{n}$ be the set gathering the FIPs such that $\Omega_{n}=\left\{\mathrm{FIP}_{1}, \mathrm{FIP}_{2}, \ldots, \mathrm{FIP}_{n}\right\}$. Let $M_{n}=$ $\max \left\{\mathrm{FIP}_{1}, \mathrm{FIP}_{2}, \ldots, \mathrm{FIP}_{n}\right\}$ be the maximum value. The probability that the latter is lower than $\sigma^{*}$ is the probability that none of the $n$ values is greater than $\sigma^{*}$, i.e.

$$
P\left(\Omega_{n}<\sigma^{*}\right)=P\left(M_{n}<\sigma^{*}\right) .
$$

According to the Fisher-Tippett-Gnedenko theorem [14], if a sequence of pairs of real numbers $\left(a_{n}, b_{n}\right)$ exists such that each $a_{n}<0$ and $\lim _{n \rightarrow+\infty} P\left(\frac{M_{n}-b_{n}}{a_{n}} \leq \sigma\right)=G(\sigma)$, where $G$ is a non-degenerate distribution function, then $G$ belongs to either the Gumbel, the Fréchet or the Weibull family. Jenkinson [13] combined the three limit distributions in a single parametric form called Generalized Extreme Value (GEV) distribution depending on a single parameter $\xi$ :

$$
G(x)=\left\{\begin{array}{l}
\exp \left(-(1+\xi x)^{-\frac{1}{\xi}}\right) \text { if } \xi \neq 0, \forall x: 1+\xi x>0, \\
\exp (-\exp (-x)) \text { if } \xi=0 .
\end{array}\right.
$$

The parameter $\xi$ is called extreme index. Its sign denotes the type of asymptotic distribution: Weibull $(\xi<0)$, Gumbel $(\xi=0)$ or Fréchet $(\xi>0)$. The variable $\frac{M_{n}-b_{n}}{a_{n}}$ is called normalised maximum of the random variable $\sigma$. The parameters $a_{n}$ and $b_{n}$ are called shape factors of the distribution. Applying this method requires a choice on the size of the extreme values population, i.e. the minimum FIP value to be considered. The larger the population, the lower the median value of the cumulative probability distribution, which is retained as the fatigue strength. The variation of the median value with the population size shows a typical shape with three sections: (i) a fast decrease as FIP values in the vicinity of the most critical nodes are included, (ii) a slowing decrease as new FIP values further from the critical nodes are considered, (iii) an asymptote as remaining FIP values are outside of the stress gradients. Depending on the EC, the decrease speed is slightly different, but we considered a unique value of $5 \%$ of the nodes to define the extreme values population, as it allows to be in the second section for all the ECs. This technique is an alternative to the volume averaging method, as it also takes into account the stress distribution surrounding stress concentrators, but does not require any assumption on the shape of the volume. It is easy to perform, computationally efficient (i.e. not time consuming) and helps to avoid computational errors due to mesh singularities. Finally, the fatigue strength $\bar{\sigma}_{D}$ (for a unit safety factor, i.e. $\mathrm{SF}=1$ ) can be expressed as

$$
\bar{\sigma}_{D}=\frac{\Sigma_{0} \times \beta}{\sigma^{*}}
$$

where $\Sigma_{0}$ is the initial applied load.

\section{Results}

\subsection{Fatigue strength of the different elementary cell topologies}

A first numerical campaign of analyses has been carried out in order to compare the fatigue strength of the different EC topologies which are summarised in Tables 1-2 for a relative density equal to $10 \%$, i.e. $\bar{\rho}=0.1$.

For the first class (Table 1), results show that BCC EC has the lowest fatigue strength in tension but the highest in shearing. Conversely, CC EC has the highest fatigue strength in tension and the lowest in shearing. These results are in agreement with the topologies of these two ECs, whose struts are essentially oriented along axes (CC) or diagonals (BCC) [4]. Also, the combination of different ECs may offer a better fatigue strength under combined tension and shear loadings (e.g. the CBCC EC which is a combination of $\mathrm{CC}$ and $\mathrm{BCC}$ ). 
Table 1: Predicted fatigue strength of the different strut-based EC topologies under the 5 loading cases of Eq. (1), for $\bar{\rho}=0.1$ and $L=3 \mathrm{~mm}$.

\begin{tabular}{|c|c|c|c|c|c|}
\hline EC & $L_{1}[\mathrm{MPa}]$ & $L_{2}[\mathrm{MPa}]$ & $L_{3}[\mathrm{MPa}]$ & $L_{4}[\mathrm{MPa}]$ & $L_{5}[\mathrm{MPa}]$ \\
\hline ACC & 10.3 & 7.2 & 7.9 & 10.3 & 7.2 \\
\hline BCC & 2 & 10.2 & 2.8 & 2.1 & 5.1 \\
\hline BFCC & 8.2 & 9.9 & 7.6 & 7.8 & 8.7 \\
\hline CBCC & 8.1 & 8.5 & 8.9 & 8.1 & 8.6 \\
\hline CBFCC & 7.6 & 9 & 8.6 & 8 & 8.7 \\
\hline CC & 18.2 & 1.7 & 2.3 & 4.8 & 1.8 \\
\hline CIC & 7.3 & 1.7 & 2.4 & 5 & 1.8 \\
\hline CFCC & 11.3 & 7.3 & 7.9 & 10.6 & 7.2 \\
\hline Diamond & 3.1 & 3.4 & 3.2 & 3.2 & 3.3 \\
\hline FCC & 9.7 & 7.4 & 7.9 & 9 & 7.6 \\
\hline FCC2+ & 8.8 & 7.2 & 7.4 & 8.2 & 7.2 \\
\hline Hexahedral & 17.4 & 1.5 & 2 & 4.3 & 1.5 \\
\hline Octahedral & 6.8 & 7.3 & 6.6 & 6.9 & 6.9 \\
\hline Octet & 12 & 10 & 8.4 & 10 & 8.6 \\
\hline Rhombic & 2.5 & 2.8 & 2.6 & 2.5 & 2.7 \\
\hline Truncated-cube & 10.7 & 5.8 & 7 & 9.6 & 6 \\
\hline Truncated-cubo & 5.1 & 2.7 & 3.2 & 4.3 & 2.8 \\
\hline Truncated-cubo2+ & 5.9 & 3.2 & 4 & 5.4 & 3.4 \\
\hline
\end{tabular}

As it clearly appears when looking at the EC topologies composed of struts, the stress concentration occurs at the intersection of struts withstanding the applied load. These localised stress concentration zones strongly affect the fatigue strength. Conversely, TPMS-based ECs show a fatigue strength higher than that characterising those made of struts, as shown in Table 2. This is due to the fact that these structures are free of connecting nodes or sharp edges.

It is noteworthy that the highly complex thin-walled TPMS are characterised by fatigue strength values that are higher than those typical of strut-based and skeletal-based cellular structures, showing, thus, a higher structural efficiency, see Table 2. Among the investigated ECs, the highest fatigue strength occurs for Gyroid configuration.

A second numerical campaign of analyses has been carried out in order to investigate the

Table 2: Predicted fatigue strength of the different skeletal TPMS and thin-walled TPMS EC topologies under the 5 loading cases of Eq. (1), for $\bar{\rho}=0.1$ and $L=3 \mathrm{~mm}$.

\begin{tabular}{|c|c|c|c|c|c|}
\hline Skeletal EC & $L_{1}[\mathrm{MPa}]$ & $L_{2}[\mathrm{MPa}]$ & $L_{3}[\mathrm{MPa}]$ & $L_{4}[\mathrm{MPa}]$ & $L_{5}[\mathrm{MPa}]$ \\
\hline BTC & 16.5 & 2.4 & 3 & 4.9 & 2.5 \\
\hline Gyroid & 7.5 & 9.9 & 8.6 & 7.7 & 9.7 \\
\hline iBCC & 12.6 & 4.1 & 5.3 & 9.7 & 4.3 \\
\hline IWP & 3.8 & 10.9 & 4.6 & 3.9 & 7.3 \\
\hline OrthoCircle & 7.6 & 3.9 & 4.3 & 5 & 4 \\
\hline Schwarz & 7.6 & 4.4 & 5.2 & 6.4 & 4.5 \\
\hline \hline Thin-walled EC & $L_{1}[\mathrm{MPa}]$ & $L_{2}[\mathrm{MPa}]$ & $L_{3}[\mathrm{MPa}]$ & $L_{4}[\mathrm{MPa}]$ & $L_{5}[\mathrm{MPa}]$ \\
\hline Batwing & 9.6 & 7.3 & 8.3 & 9.3 & 7.5 \\
\hline BTC & 7.7 & 4 & 5.3 & 7.2 & 4.2 \\
\hline F-RD & 9.9 & 7.2 & 8.5 & 9.5 & 7.5 \\
\hline Gyroid & 16.3 & 11.5 & 12.3 & 14.6 & 11.4 \\
\hline IWP & 14.7 & 9 & 10.3 & 13.1 & 9.2 \\
\hline Manta & 12.9 & 11.5 & 11.8 & 12.6 & 11.4 \\
\hline Neovius & 13.9 & 4.4 & 6.1 & 10.6 & 4.7 \\
\hline Schone-I6 & 7.9 & 6.9 & 7.2 & 7.6 & 7.4 \\
\hline Schwarz & 8.3 & 6.4 & 7.1 & 8.2 & 6.5 \\
\hline
\end{tabular}

influence of the EC relative density on its fatigue strength. For the sake of brevity, only the results of two types of unit cells, namely Octet and thin-walled Gyroid, are presented and 


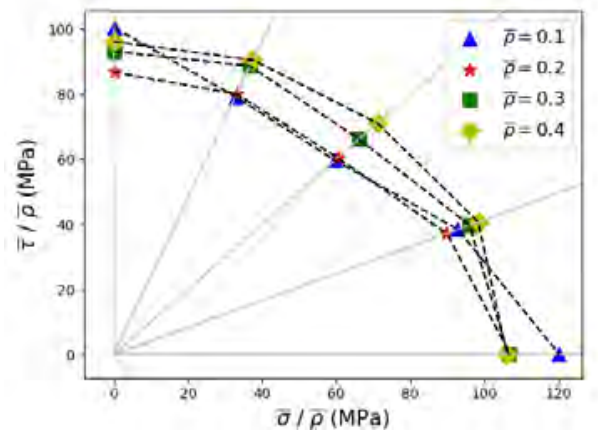

(a) Octet EC

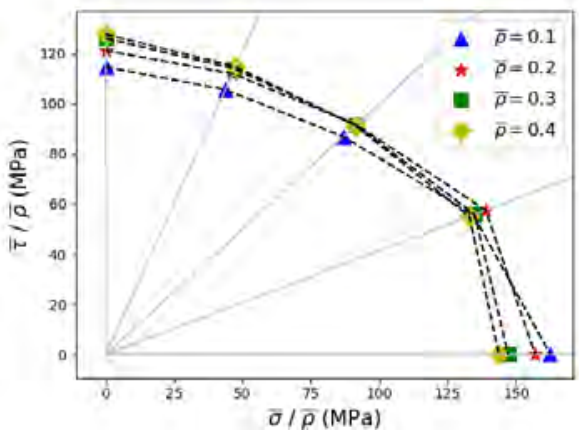

(b) Gyroid EC

Fig. 5. Density variation effects on the normalised fatigue strength.

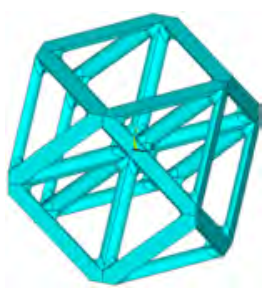

(a)

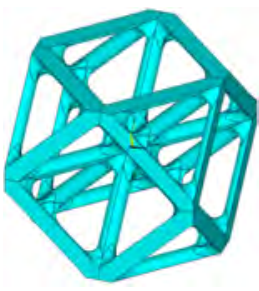

(b)

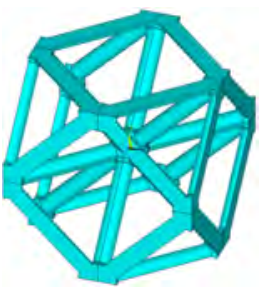

(c)

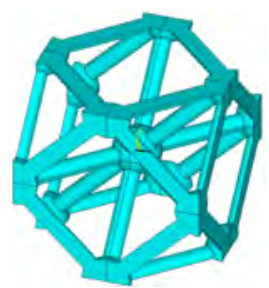

(d)

Fig. 6. Octet EC with a: fillet radius $R=\frac{1}{3} d_{C A D}$, b: fillet radius $R=\frac{2}{3} d_{C A D}$, c: spherical nodes of diameter $\Omega=2.4 \times d_{C A D}$ and d: spherical nodes of diameter $\Omega=2.8 \times d_{C A D}$.

discussed. In Fig. 5, the trend of the fatigue strength vs. the relative density is illustrated. For each case, the relative density varies between 0.1 and 0.4 which are classical values taken from literature. As expected, the higher the relative density, the higher the fatigue strength. Moreover, the fatigue strength curves normalised with the relative density are well represented by a single power law for each type of unit cell.

\subsection{Suppression of stress concentration zones: the effect of spherical nodes and fillet radii on the lattice fatigue strength}

The presence of stress concentrations due to sharp corners in the lattice structures are the cause of the underestimation of the fatigue stress (and fatigue life). For the sake of brevity, this effect is discussed in the followings only for the Octet EC. An illustration of the latter with spherical nodes as well as fillet radii joining the elementary struts is given in Fig. 6 for different values of the sphere diameter $\Omega$ and the fillet radii $R$.

In this picture, the sphere diameter and the fillet radii are expressed as a function of the strut diameter, i.e. $\Omega=\delta d_{C A D}$ and $R=\gamma d_{C A D}$. As it can be easily inferred from Fig. 7a, the presence of a fillet radius joining concurrent struts allows for increasing the fatigue strength of the lattice. Moreover, the greater the single fillet radius the greater the relative density. As illustrated in Fig. 7b, for standard values of the fillet radius (i.e. values that can properly be 


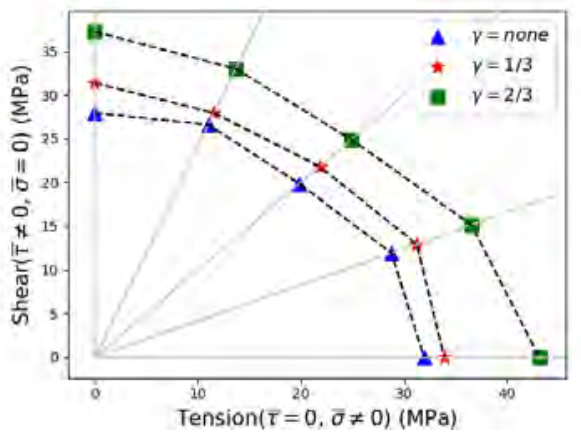

(a) Absolute

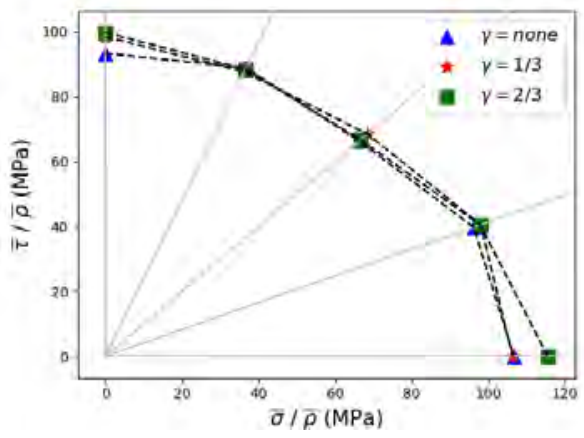

(b) Normalised

Fig. 7. Influence of the fillet radius on the predicted fatigue strength for Octet $\mathrm{EC}$ and $d_{C A D}=$ $0.52 \mathrm{~mm}$.

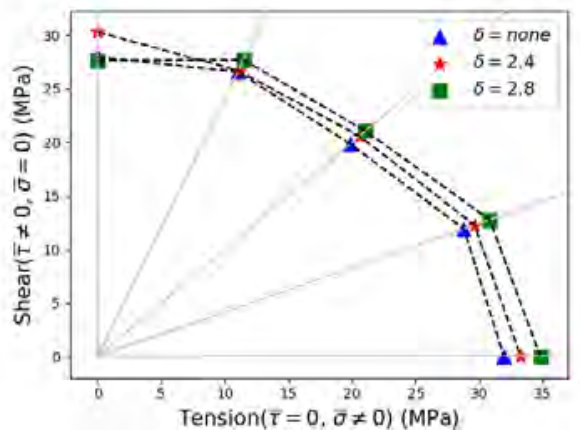

(a) Absolute

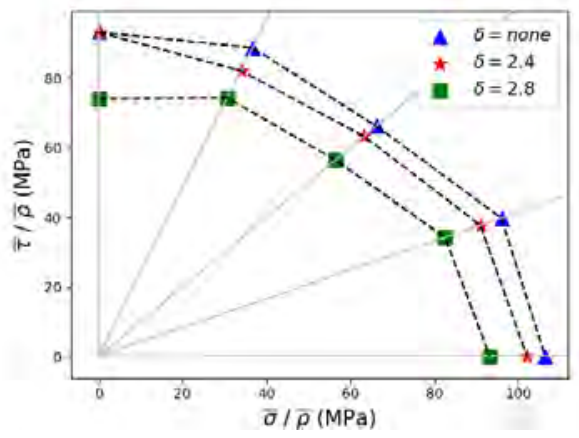

(b) Normalised

Fig. 8. Influence of the sphere diameter on the predicted fatigue strength for Octet EC and $d_{C A D}=0.52 \mathrm{~mm}$.

obtained by means of SLM process) the fatigue strength always grows almost as fast as the relative density. Accordingly, a lattice characterised by fillet radius is always a lightweight structure. An analysis of Fig. 8a suggests that the presence of spherical nodes between struts leads to an overall enhancement of the fatigue strength of the lattice. However, this increment is lower than that obtained for the solution characterised by fillet radii. However, the greater the sphere diameter the greater the relative density is. As illustrated in Fig. 8b, the relative density grows faster than the fatigue strength: accordingly, the strength-to-weight ratio of the lattice reduces.

\section{Conclusions}

A high-cycle fatigue criterion based on the distribution of extreme values of the Crossland equivalent stress has been developed in order to investigate the influence of the EC topology 
on the fatigue strength of several classes of lattice structures. Results proved that the thinwalled TPMS lattices exhibit superior fatigue strength when compared to strut-based and skeletal-based lattice structures, showing higher structural efficiency. It is also observed that the fatigue strength is proportional to the relative density varying from 0.1 to 0.4 .

The stiffest cell types identified were the Octet for strut-based lattice structures and thinwalled Gyroid for TPMS. Therefore, these cell types were chosen for manufacture and fatigue testing.

\section{References}

1. S. Amin Yavari, S. Ahmadi, R. Wauthle, B. Pouran, J. Schrooten, H. Weinans, A. Zadpoor, Relationship between unit cell type and porosity and the fatigue behavior of selective laser melted meta-biomaterials, J Mech Behav Biomed Mater, 43: 91-100 (2015)

2. S. Zhao, S. Li, W. Hou, Y. Hao, R. Yang, R. Misra, The influence of cell morphology on the compressive fatigue behavior of ti-6al-4v meshes fabricated by electron beam melting, J Mech Behav Biomed Mater, 59: 251-264 (2016)

3. R. Hedayati, H. Hosseini-Toudeshky, M. Sadighi, M. Mohammadi-Aghdam, A. Zadpoor, Computational prediction of the fatigue behavior of additively manufactured porous metallic biomaterials, Int J Fatigue, 84: 67-79 (2016)

4. K. Refai, M. Montemurro, C. Brugger, N. Saintier, Determination of the effective elastic properties of titanium lattice structures, Mech Adv Mater Struc.

URL https://doi.org/10.1080/15376494.2018.1536816

5. S. KapferaStephen, T. Hyde, K. Mecke, H. Arns, E. Schröder-Turk, Minimal surface scaffold designs for tissue engineering, Biomaterials, 32: 6875-6882 (2011)

6. E.J. Barbero, Finite element analysis of composite materials (CRC Press, Taylor \& Francis Group, 2008)

7. M. Montemurro, A. Catapano, D. Doroszewski, A multi-scale approach for the simultaneous shape and material optimisation of sandwich panels with cellular core, Compos Part B-Eng, 91: 458-472 (2016)

8. L. Cappelli, M. Montemurro, F. Dau, L. Guillaumat, Characterisation of composite elastic properties by means of a multi-scale two-level inverse approach, Compos Struct, 204: 767777 (2018)

9. B. Crossland, Effect of large hydrostatic pressures on the torsional fatigue strength of an alloy steel, In: Proceedings of the International Conference on Fatigue of Metals, Inst Mech Eng, London, p.138-149 (1956)

10. K. Dang Van, Sur la résistance à la fatigue des métaux, In: Sciences Technique Armement, 47: 641-722 (1973)

11. A. Banvillet, T. Palin-Luc, S. Lasserre, A volumetric energy based high cycle multiaxial fatigue citerion, Int J Fatigue, 25: 755-769 (2003)

12. B. Vayssette, N. Saintier, C. Brugger, M. Elmay, E. Pessard, Surface roughness of Ti6Al-4V parts obtained by SLM and EBM: Effect on the High Cycle Fatigue life, Procedia Eng, 213: 89-97 (2018)

13. A. F. Jenkinson. The frequency distribution of the annual maximum (or minimum) values of meteorological elements. In: Quart J Roy Meteorol Soc, 81.348: 158-171 (1955)

14. R. A. Fisher, The Genetical Theory of Natural Selection (Oxford University Press, 1930) 